\title{
CROSSED PRODUCTS OF HILBERT C*-BIMODULES BY BUNDLES
}

\author{
TSUYOSHI KAJIWARA and YASUO WATATANI
}

(Received 20 December 1996; revised 11 April 1997)

Communicated by G. Robertson

\begin{abstract}
We present the definition of crossed products of Hilbert $\mathrm{C}^{*}$-bimodules by Hilbert bundles with commuting finite group actions and finite dimensional fibers. This is a general construction containing the bundle construction and crossed products of Hilbert $\mathrm{C}^{*}$-bimodule by finite groups. We also study the structure of endomorphism algebras of the tensor products of bimodules. We also define the multiple crossed products using three bimodules containing more than 2 bundles and show the associativity law. Moreover, we present some examples of crossed product bimodules easily computed by our method.
\end{abstract}

1991 Mathematics subject classification (Amer. Math. Soc.): primary 46L35,46L55.

\section{Introduction}

Jones $[\mathrm{J}]$ initiated the index theory of subfactors. The notion of bimodules in subfactor theory was introduced by Ocneanu [O], and is a very powerful tool for studying index theory of $\mathrm{W}^{*}$-algebras. Following Ocneanu, Yamagami developed the study of the categorical structure of $\mathrm{W}^{*}$-bimodules ([Y1, Y2, Y3]). The notion of Hilbert bundle over a countable discrete group with two-sided commuting finite group actions and finite dimensional fibers was introduced by Kosaki and Yamagami [KoY], and is a very convenient tool for constructing many examples of bimodules of finite index with various combinatorial structures. In [KaY], the first named author and Yamagami extended this construction to the case of compact group actions on von Neumann algebras.

On the other hand, the second named author [W] initiated index theory in $\mathrm{C}^{*}$ algebras. In [KW1], we define the notion of Hilbert $\mathrm{C}^{*}$-bimodules of finite type for studying $\mathrm{C}^{*}$-index theory using bimodules and $\mathrm{K}$-theory. Hilbert $\mathrm{C}^{*}$-bimodules of

(C) 1998 Australian Mathematical Society $0263-6115 / 98 \$ A 2.00+0.00$ 
finite type are considered the $\mathrm{C}^{*}$-version of $W^{*}$-bimodules with finite index.

We also give in [KW1] the bundle construction of a Hilbert $\mathrm{C}^{*}$-bimodule corresponding to the $\mathrm{W}^{*}$ situation [KoY]. In [KW2], we give the crossed product construction of Hilbert $\mathrm{C}^{*}$-bimodules by countable discrete groups, and present some examples. This is also considered to be a generalization of the notion of crossed product imprimitivity bimodules given by [CMW, $\mathrm{Co}, \mathrm{K}]$.

In this paper, we give a construction simultaneously generalizing the above two ones. We give the crossed product construction of a Hilbert $\mathrm{C}^{*}$-bimodule by a bundle with commuting finite groups, and present some examples which are computed easily using our method of multiple crossed products.

In Section 2, we record the definitions and fundamental matters concerning Hilbert $\mathrm{C}^{*}$-bimodule of finite type, and define the crossed products of Hilbert $\mathrm{C}^{*}$-bimodules by bundles. We also study categorical structures of crossed product bimodules.

In Section 3, we define the crossed products of bundles by a bundle by the method similar to that of Section 2. We also define a crossed product construction for three objects, and show the associativity law of crossed products. It is natural to call this construction 'multiple crossed products'. Using this associativity law, we may make computations for many bimodules in the simplest settings.

In Section 4, we present some examples for which we may use the technique in Section 3 for the computation. In Example 2, we consider a generalization of duality between type $A$ and type D presented in [KW2] and calculate generators of bimodules appearing in each floor and their inclusions explicitly. In Example 3, we present a construction of bimodules which generate Kac algebras naturally using our method.

The authors express their hearty thanks to S. Yamagami for several discussions and sending us new preprints about these matters, and the referee for careful reading and many valuable comments.

\section{Crossed products by bundles}

We review the definition of Hilbert $\mathrm{C}^{*}$-bimodules of finite type following [KW1]. We refer to $[\mathrm{B}]$ for the definition of Hilbert $\mathrm{C}^{*}$-module. Let $A$ and $B$ be unital $\mathrm{C}^{*}$-algebras. Let $X$ be a $\mathbb{C}$-vector space.

DEFINITION 1 ([KW1]). $X$ is called a Hilbert $C^{*}$-bimodule (or Hilbert A-B bimod$u l e$ ) if the following conditions hold.

(1) $X$ is a left Hilbert $A$-module.

(2) $X$ is a right Hilbert $B$-module.

(3) Left $A$ action and right $B$ action commute with each other.

(4) Let $\lambda(a) x=a x, \rho(b) x=x b$ for $a \in A, b \in B$ and $x \in X$. Then $\lambda(a)$ is bounded and has an adjoint with respect to $\langle,\rangle_{B}$, and $\rho(b)$ is bounded and has an 
adjoint with respect to ${ }_{A}\langle$,$\rangle .$

(5) The two norms on $X$ given by ${ }_{A}\|x\|=\|\|_{A}\langle x, x\rangle \|^{1 / 2}$ and $\|x\|_{B}=\left\|\langle x, x\rangle_{B}\right\|^{1 / 2}$ are equivalent (Pimsner-Popa type inequality).

DEFINITION 2 ([KW1]). A Hilbert $A-B$ bimodule $X$ is said to be of finite type if the following two conditions hold.

(1) There exists a finite subset $\left\{u_{i}\right\}_{i}$ in $X$ such that $\sum_{i} u_{i}\left\langle u_{i}, x\right\rangle_{B}=x$ for all $x \in X$.

(2) There exists a finite subset $\left\{v_{j}\right\}_{j}$ in $X$ such that $\sum_{j}{ }_{A}\left\langle x, v_{j}\right\rangle v_{j}=x$ for all $x \in X$.

We call this finite subset $\left\{u_{i}\right\}_{i}$ a right $B$-basis of $X$ and $\left\{v_{j}\right\}_{j}$ a left A-basis of $X$. Moreover we denote the right index and left index of $X$ by $\mathrm{r}$-Ind $[X]=\sum_{i}\left\langle u_{i}, u_{i}\right\rangle$ and $1-\operatorname{Ind}[X]=\sum_{j}\left\langle v_{j}, v_{j}\right\rangle_{B}$.

The following lemma is convenient for verifying the axioms of Hilbert $\mathrm{C}^{*}$-bimodules, because analytic properties follow from purely algebraic properties under the existence of bases.

LEMMA 3 ([KW1]). Let a complex vector space $X$ satisfy the following (1)-(10).

(1) $X$ is a left A-module.

(2) X has a left self-adjoint (not necessarily positive) $A$-inner product ${ }_{A}\langle\cdot, \cdot\rangle$.

(3) ${ }_{A}\langle a x, y\rangle=a_{A}\langle x, y\rangle$.

(4) $X$ is a right $B$-module.

(5) $X$ has a right self-adjoint (not necessarily positive) $B$-inner product $\langle\cdot, \cdot\rangle_{B}$.

(6) $\langle x, y b\rangle_{B}=\langle x, y\rangle_{B} b$.

(7) The left $A$ action and right $B$ action commute with each other.

(8) $\langle a x, y\rangle_{B}=\left\langle x, a^{*} y\right\rangle_{B}$ and ${ }_{A}\langle x, y b\rangle={ }_{A}\left\langle x b^{*}, y\right\rangle$.

(9) There exists a finite subset $\left\{u_{i}\right\}_{i}$ in $X$ such that $\sum_{i} u_{i}\left\langle u_{i}, x\right\rangle_{B}=x$ for all $x \in X$.

(10) There exists a finite subset $\left\{v_{j}\right\}_{j}$ in $X$ such that $\sum_{j}{ }_{A}\left\langle x, v_{j}\right\rangle v_{j}=x$ for all $x \in X$.

Then the other properties in Definition 1.1 are automatically satisfied, and $X$ becomes a Hilbert $C^{*}$-bimodule of finite type.

We denote by ${ }_{A} \operatorname{End}_{B}(X)$ the elements in $\mathbb{C}_{\mathbb{C}} \operatorname{End}_{\mathbb{C}}(X)$ which commute with left $A$, right $B$ actions. When $X$ is of finite type $T \in{ }_{A} \operatorname{End}_{B}(X)$ has adjoints with respect to both inner products and is bounded [KW1].

Let $G$ be a countable discrete group, $H$ and $K$ finite subgroups of $G$. We recall the definition of $H-K$ bundle from [KoY].

DEFINITION 4 ([KoY]). A finite-dimensional Hilbert space $V$ admitting a left $H$ right $K$ action is called an $H-K$ bundle over $G$ if the following are satisfied.

(1) $V=\bigoplus_{g \in G} V_{g}$, where $V_{g}$ is also a finite dimensional Hilbert space.

(2) For $h \in H$ and $k \in K,\langle h \cdot x, h \cdot y\rangle=\langle x, y\rangle$ and $\langle x \cdot k, y \cdot k\rangle=\langle x, y\rangle$ for $x$, $y \in V$. 
(3) For $h \in H, h \cdot V_{g} \subset V_{h g}$ and for $k \in K, V_{g} \cdot k \subset V_{g h}$.

We call $\left\{g \in G: V_{g} \neq 0\right\}$ the support of $V$. We denote by EndV $(V)$ (more precisely $\left.{ }_{H} \operatorname{EndV}_{K}(V)\right)$ the elements in ${ }_{C} \operatorname{End}_{\mathbb{C}}(V)$ which commute with left $H$, right $K$ actions and the diagonal representation of $C(G)$.

DEFINITION 5 ([KoY]). The conjugate bundle $\bar{V}$ of $V$ is defined as follows.

(1) As a space, $\bar{V}$ is the direct sum of finite-dimensional Hilbert spaces $\bar{V}_{g^{-1}}$, where each $\bar{V}_{g^{-1}}$ is the conjugate Hilbert space of $V_{g}$. We use the notation $\bar{v}_{g^{-1}}$ when $v_{g}$ is considered as an element of $\bar{V}_{g^{-1}}$.

(2) $\bar{V}$ is a left $K$-right $H$ bundle, where the two actions are defined as follows: $\bar{v} \cdot h=\overline{h^{-1} \cdot v}, k \cdot \bar{v}=\overline{v \cdot k^{-1}}$, where $\bar{v}$ is $v$ considered as an element in $\bar{V}$.

Let $X$ be a Hilbert $A$ - $B$ bimodule of finite type, $\alpha$ be an action of $G$ on $A, \beta$ be an action of $G$ on $B$ and $\gamma$ be a homomorphism from $G$ to the isometry group of $X$.

DEFINITION 6 ([KW2]). The system $(X, A, B, \gamma, \alpha, \beta, G)$ is called a $G$-equivariant system of bimodules if the following are satisfied for each $g \in G$.

$$
\begin{aligned}
& \alpha_{g}\left({ }_{A}\langle x, y\rangle\right)={ }_{A}\left\langle\gamma_{g}(x), \gamma_{g}(y)\right\rangle, \quad \gamma_{g}(a x)=\alpha_{g}(a) \gamma_{g}(x), \\
& \beta_{g}\left(\langle x, y\rangle_{B}\right)=\left\langle\gamma_{g}(x), \gamma_{g}(y)\right\rangle_{B}, \quad \gamma_{g}(x b)=\gamma_{g}(x) \beta_{g}(b) \text {, }
\end{aligned}
$$

for $x, y \in X, a \in A, b \in B$.

DEFINITION 7. Let $V$ be an $H-K$ bundle over $\Gamma .(X, V)$ is called a covariant system of bimodules if ( $X, A, B, \gamma, \alpha, \beta, \Gamma$ ) is a $\Gamma$-equivariant system of bimodules.

We denote by $\hat{V}$ the tensor product $X \otimes V$ considered as only a vector space. We write a monomial element of $\hat{V}$ in the form $x \otimes v_{g} \lambda_{g}\left(x \in X, v_{g} \in V_{g}\right)$, where $\lambda_{g}$ is only a symbol representing the position of $v_{g}$ in V. When $X={ }_{A} A_{A}$ and $V={ }_{G} C(G)_{G}$, we denote $a \otimes 1 \lambda_{g}$ by $a \lambda_{g}$, adapting to the convention of crossed product $C^{*}$-algebras.

We define two-sided actions of $A \rtimes_{\alpha} H$ and $B \rtimes_{\beta} K$ on $\hat{V}$ as follows.

$$
\begin{aligned}
& a\left(x \otimes v_{g} \lambda_{g}\right)=(a x) \otimes v_{g} \lambda_{g}, \quad h\left(x \otimes v_{g} \lambda_{g}\right)=\gamma_{h}(x) \otimes h\left(v_{g}\right) \lambda_{h g}, \\
& \left(x \otimes v_{g} \lambda_{g}\right) b=x \beta_{g}(b) \otimes v_{g} \lambda_{g}, \quad\left(x \otimes v_{g} \lambda_{g}\right) k=x \otimes\left(v_{g}\right) k \lambda_{g k} .
\end{aligned}
$$

We remark that ${ }_{A}\left(X \otimes V_{g}\right)_{B}$ is isomorphic to $\operatorname{dim}\left(V_{g}\right)_{A}(X g)_{B}$ where $X g$ is the $A-B$ bimodule twisting the right $B$ action by $\beta_{g}$.

We define two-sided inner products as follows. 


$$
\begin{aligned}
& A \rtimes_{G} H\left\langle x \otimes v_{g} \lambda_{g}, x^{\prime} \otimes v_{g^{\prime}}^{\prime} \lambda_{g^{\prime}}\right\rangle \\
& = \begin{cases}{ }_{A}\left\langle x, \gamma_{h}\left(x^{\prime}\right)\right\rangle\left\langle v_{g}, h\left(v_{g^{\prime}}^{\prime}\right)\right\rangle \lambda_{h} & \text { if } g g^{\prime-1}=h \in H \\
0 & \text { otherwise; }\end{cases}
\end{aligned}
$$

$\left\langle x \otimes v_{g} \lambda_{g}, x^{\prime} \otimes v_{g^{\prime}}^{\prime} \lambda_{g^{\prime}}\right\rangle_{B \rtimes_{\beta} K}$

$$
= \begin{cases}\left.\beta_{g}{ }^{\prime}\left(\left\langle x, x^{\prime}\right\rangle_{B}\right)\left\langle v_{g},\left(v_{g^{\prime}}^{\prime}\right) k^{-1}\right)\right\rangle \lambda_{k} & \text { if } g^{-1} g^{\prime}=k \in K \\ 0 & \text { otherwise. }\end{cases}
$$

When $X=A=B=\mathbb{C}$, these formulas give left $C^{*}(H)$-right $C^{*}(K)$ inner products.

PROPOSITION 8. This system satisfies the conditions (1)-(8) in Lemma 3.

PROOF. We prove only (2), (3), (6) and (8).

We show (2). The range of the inner product is clearly total in $A$. We assume $g g^{\prime-1}=h \in H$.

$$
\begin{aligned}
\left({ }_{A \rtimes_{\alpha} H}\left\langle x \otimes v_{g} \lambda_{g}, x^{\prime} \otimes v_{g^{\prime}} \lambda_{g^{\prime}}\right\rangle\right)^{*} & =\left({ }_{A}\left\langle x, \gamma_{h}\left(x^{\prime}\right)\right\rangle\left\langle v_{g}, h\left(v_{g^{\prime}}^{\prime}\right) \lambda_{h}\right)^{*}\right. \\
& =\lambda_{h^{-1}}\left\langle\gamma_{h}\left(x^{\prime}\right), x\right\rangle \overline{\left\langle v_{g}, h\left(v_{g^{\prime}}^{\prime}\right)\right\rangle} \\
& ={ }_{A}\left\langle x^{\prime}, \gamma_{h^{-1}}(x)\right\rangle\left\langle v_{g^{\prime}}^{\prime}, h^{-1}\left(v_{g}\right)\right\rangle \lambda_{h^{-1}} \\
& ={ }_{A \rtimes_{\alpha} H}\left\langle x^{\prime} \otimes v_{g^{\prime}}^{\prime} \lambda_{g^{\prime}}, x \otimes v_{g} \lambda_{g}\right\rangle .
\end{aligned}
$$

We show (3). We assume $g^{-1} g^{\prime}=h \in H$.

$$
\begin{aligned}
A \rtimes_{\alpha} H\left\langle a\left(x \otimes v_{g} \lambda_{g}\right), x^{\prime} \otimes v_{g^{\prime}}^{\prime} \lambda_{g^{\prime}}\right\rangle & ={ }_{A}\left\langle a x, \gamma_{h}\left(x^{\prime}\right)\right\rangle\left\langle v_{g}, h\left(v_{g^{\prime}}^{\prime}\right)\right\rangle \lambda_{h} \\
& =a_{A}\left\langle x \otimes v_{g} \lambda_{g}, x^{\prime} \otimes v_{g^{\prime}}^{\prime} \lambda_{g^{\prime}}\right\rangle \\
A \rtimes_{\alpha} H\left\langle h^{\prime}\left(x \otimes v_{g} \lambda_{g}\right), x^{\prime} \otimes v_{g^{\prime}}^{\prime} \lambda_{g^{\prime}}\right\rangle & ={ }_{A \rtimes_{\alpha} H}\left\langle\gamma_{h^{\prime}}(x) \otimes h^{\prime}\left(v_{g}\right) \lambda_{h^{\prime} g}, x^{\prime} \otimes v_{g^{\prime}}^{\prime} \lambda_{g^{\prime}}\right\rangle \\
& ={ }_{A}\left\langle\gamma_{h^{\prime}}(x), \gamma_{h^{\prime} h}\left(x^{\prime}\right)\right\rangle\left\langle h^{\prime}\left(v_{g}\right), h^{\prime} h\left(v_{g^{\prime}}^{\prime}\right)\right\rangle \lambda_{h^{\prime} h} \\
& =\alpha_{h^{\prime}}\left({ }_{A}\left\langle x, \gamma_{h}\left(x^{\prime}\right)\right\rangle\right)\left\langle v_{g}, h\left(v_{g^{\prime}}^{\prime}\right)\right\rangle \lambda_{h^{\prime} h} \\
& =\lambda_{h^{\prime}}\left(A \rtimes_{a} H\left\langle x \otimes v_{g} \lambda_{g}, x^{\prime} \otimes v_{g^{\prime}}^{\prime} \lambda_{g^{\prime}}\right\rangle\right) .
\end{aligned}
$$

We show (6). We assume $g^{-1} g^{\prime}=k \in K$.

$$
\begin{aligned}
\left\langle x \otimes v_{g} \lambda_{g},\left(x^{\prime} \otimes v_{g^{\prime}}^{\prime} \lambda_{g^{\prime}}\right) k^{\prime}\right\rangle_{B \rtimes_{\beta} K} & =\left\langle x \otimes v_{g} \lambda_{g}, x^{\prime} \otimes\left(v_{g^{\prime}}^{\prime}\right) k^{\prime} \lambda_{g^{\prime} k^{\prime}}\right\rangle_{B \rtimes_{\sharp} K} \\
& =\beta_{g^{-1}}\left(\left\langle x, x^{\prime}\right\rangle_{B}\right)\left\langle v_{g},\left(v_{g^{\prime}}^{\prime}\right) k^{\prime} k^{\prime-1} k^{-1}\right\rangle \lambda_{k k^{\prime}} \\
& =\beta_{g^{-1}}\left(\left\langle x, x^{\prime}\right\rangle_{B}\right)\left\langle v_{g},\left(v_{g^{\prime}}^{\prime}\right) k^{-1}\right\rangle \lambda_{k} \lambda_{k^{\prime}} \\
& =\left(\left\langle x \otimes v_{g} \lambda_{g}, x^{\prime} \otimes v_{g^{\prime}}^{\prime} \lambda_{g^{\prime}}\right\rangle_{B \rtimes_{\beta} K}\right) \lambda_{k^{\prime}} .
\end{aligned}
$$


We assume $g^{-1} g^{\prime}=k \in K$.

$$
\begin{aligned}
\left\langle x \otimes v_{g} \lambda_{g},\left(x^{\prime} \otimes v_{g^{\prime}}^{\prime} \lambda_{g^{\prime}}\right) b\right\rangle_{B \rtimes_{\beta} K} & =\left\langle x \otimes v_{g} \lambda_{g}, x^{\prime} \beta_{g^{\prime}}(b) \otimes v_{g^{\prime}}^{\prime} \lambda_{g^{\prime}}\right\rangle_{B \rtimes_{\beta} K} \\
& =\beta_{g^{-1}}\left(\left\langle x, x^{\prime} \beta_{g^{\prime}}(b)\right\rangle_{B}\right)\left\langle v_{g},\left(v_{g^{\prime}}^{\prime}\right) k^{-1}\right\rangle \lambda_{k} \\
& \left.=\beta_{g^{-1}}\left(\left\langle x, x^{\prime}\right\rangle_{B} \beta_{g^{\prime}}(b)\right)\right\rangle\left\langle v_{g},\left(v_{g^{\prime}}^{\prime}\right) k^{-1}\right\rangle \lambda_{k} \\
& =\beta_{g^{-1}}\left(\left\langle x, x^{\prime}\right\rangle_{B}\right) \beta_{k^{\prime}}(b)\left\langle v,\left(v_{g^{\prime}}^{\prime}\right) k^{-1}\right\rangle \lambda_{k} \\
& =\left\langle x \otimes v_{g} \lambda_{g}, x^{\prime} \otimes v_{g^{\prime}}^{\prime} \lambda_{g^{\prime}}\right\rangle_{B \rtimes_{\beta} K} b
\end{aligned}
$$

when $g^{-1} g^{\prime}=k \in K$ and is otherwise zero.

We show (8). We assume $g^{-1} g^{\prime}=k \in K$.

$$
\begin{aligned}
\left\langle a\left(x \otimes v_{g} \lambda_{g}\right), x^{\prime} \otimes v_{g^{\prime}}^{\prime} \lambda_{g^{\prime}}\right\rangle_{B \rtimes_{\beta} K} & =\beta_{g^{-1}}\left(\left\langle a x, x^{\prime}\right\rangle_{B}\right)\left(\left\langle v_{g},\left(v_{g^{\prime}}^{\prime}\right) k^{-1}\right\rangle \lambda_{k}\right. \\
& =\beta_{g^{-1}}\left(\left\langle x, a^{*} x^{\prime}\right\rangle_{B}\right)\left\langle v_{g},\left(v_{g^{\prime}}^{\prime}\right) k^{-1}\right\rangle \lambda_{k} \\
& =\left\langle x \otimes v_{g} \lambda_{g}, a^{*}\left(x^{\prime} \otimes v_{g^{\prime}}^{\prime} \lambda_{g}\right)\right\rangle_{B \rtimes_{B} K} .
\end{aligned}
$$

We assume $(h g)^{-1} g^{\prime}=k \in K$ :

$$
\begin{aligned}
\left\langle h\left(x \otimes v_{g} \lambda_{g}\right), x^{\prime} \otimes v_{g^{\prime}}^{\prime}, \lambda_{g^{\prime}}\right\rangle_{B \rtimes_{\beta} K} & =\left\langle\gamma_{h}(x) \otimes h\left(v_{g}\right) \lambda_{h g}, x^{\prime} \otimes v_{g}^{\prime}, \lambda_{g^{\prime}}\right\rangle_{B \rtimes_{\beta} K} \\
& =\beta_{g^{-1} h^{-1}}\left(\left\langle\gamma_{h}(x), x\right\rangle_{B}\right)\left\langle h\left(v_{g}\right),\left(v_{g^{\prime}}^{\prime}\right) k^{-1}\right\rangle \lambda_{k} \\
& =\beta_{g^{-1}}\left(\left\langle x, \gamma_{h^{-1}}\left(x^{\prime}\right)\right\rangle_{B}\right)\left\langle h\left(v_{g}\right),\left(v_{g^{\prime}}^{\prime}\right) k^{-1}\right\rangle \lambda_{k} \\
& =\beta_{g^{-1}}\left(\left\langle x, \gamma_{h^{-1}}\left(x^{\prime}\right)\right\rangle_{B}\right)\left\langle v_{g}, h^{-1}\left(v_{g^{\prime}}^{\prime}\right) k^{-1}\right\rangle \lambda_{k} \\
& =\left\langle x \otimes v_{g} \lambda_{g}, \gamma_{h^{-1}}\left(x^{\prime}\right) \otimes h^{-1}\left(v_{g^{\prime}}^{\prime}\right) \lambda_{h^{-1} g^{\prime}}\right\rangle_{B \rtimes_{\beta} K} \\
& =\left\langle x \otimes v_{g} \lambda_{g}, h^{-1}\left(x^{\prime} \otimes v_{g^{\prime}}^{\prime} \lambda_{g^{\prime}}\right)\right\rangle_{B \rtimes_{\beta} K}
\end{aligned}
$$

and is otherwise equal to 0 .

Other properties are proved similarly.

Let $\Sigma$ be the support of $V,\left\{\sigma_{m}\right\}_{m}$ be a subset of $\Sigma$ which meets each $K$-orbit only once, and $\left\{v_{l}^{m}\right\}_{l}$ be a complete orthonormal system (CONS) in $V_{\sigma_{m}}$. We put $q_{m, l}=v_{l}^{m} \lambda_{\sigma_{m}}$. Similarly, let $\left\{\tau_{n}\right\}_{n}$ be a similar subset concerning $H$-action on $\Sigma$, and $\left\{v_{k}^{n}\right\}_{k, n}$ be a CONS in $V_{\tau_{n}}$. We put $p_{n, k}=v_{k}^{n} \lambda_{\tau_{n}}$.

Let $\left\{u_{i}\right\}_{i}$ be a right $B$-basis of $X$ and $\left\{v_{j}\right\}_{j}$ be a left $A$-basis of $X$.

Proposition 9. The family $\left\{u_{i} \otimes q_{m, l}\right\}_{i, m . l}$ constitutes a right $B \rtimes_{\beta} K$-basis for $\hat{V}$ and $\left\{v_{j} \otimes p_{n . k}\right\}_{j, n . k}$ constitutes a left $A \rtimes_{\alpha} H$-basis for $\hat{V}$.

PROOF. Let $\sigma_{m^{\prime}}=g^{\prime}$, with $g^{\prime}$ in the same right $K$ coset as $g$, and let $k$ be $g^{\prime-1} g$. 


$$
\begin{aligned}
\sum_{i . m . l} u_{i} & \otimes q_{m . l}\left\langle u_{i} \otimes q_{m . l}, x \otimes v_{g} \lambda_{g}\right\rangle_{B \rtimes_{\beta} K} \\
& =\sum_{i . l}\left(u_{i} \otimes v_{l}^{m^{\prime}} \lambda_{\sigma_{m^{\prime}}}\right) \beta_{g^{\prime-1}}\left(\left\langle u_{i}, x\right\rangle_{B}\right)\left\langle v_{l}^{m^{\prime}},\left(v_{g}\right) k^{-l}\right\rangle \lambda_{k} \\
& =\sum_{i} u_{i}\left\langle u_{i}, x\right\rangle_{B} \otimes\left(\sum_{l}\left(v_{l}^{m^{\prime}}\right) k\left\langle v_{l}^{m^{\prime}},\left(v_{g}\right) k^{-1}\right\rangle\right) \lambda_{g^{\prime} k} \\
& =\sum_{i} u_{i}\left\langle u_{i}, x\right\rangle_{B} \otimes \sum_{l}\left(v_{l}^{m^{\prime}}\left\langle v_{l}^{m^{\prime}},\left(v_{g}\right) k^{-1}\right\rangle\right) k \\
& =x \otimes v_{g} \lambda_{g} .
\end{aligned}
$$

We have used the properties of bases and CONS in Hilbert spaces.

The proof is similar for the left basis.

PROPOSITION 10. $\hat{V}$ is made into a Hilbert $C^{*}$-bimodule of finite type, and we have $\mathrm{r}-\operatorname{Ind}[\hat{V}]=\mathrm{r}-\operatorname{Ind}[X]\left(\sum_{m} \operatorname{dim}\left(V_{\sigma_{m}}\right)\right), 1-\operatorname{Ind}[\hat{V}]=1-\operatorname{Ind}[X]\left(\sum_{m} \operatorname{dim}\left(V_{\tau_{n}}\right)\right)$. Moreover, we have $\operatorname{Ind}\left[X \rtimes_{\gamma} V\right]=\operatorname{Ind}[X]\left(\sum_{m} \operatorname{dim}\left(V_{\sigma_{m}}\right)\right)\left(\sum_{n} \operatorname{dim}\left(V_{\tau_{n}}\right)\right)$.

ProOF. The first statement follows from Proposition 9 and Lemma 3.

We prove the second statement only for the right index. We take a right basis $\left\{u_{i} \otimes q_{m, l}\right\}_{i, m, l}$ as in Proposition 9.

$$
\begin{aligned}
\mathrm{r}-\mathrm{Ind}\left[X \rtimes_{\gamma} V\right] & =\sum_{i, m, l} A{\rtimes_{\alpha} H}\left\langle u_{i} \otimes q_{m . l}, u_{i} \otimes q_{m, l}\right\rangle \\
& =\sum_{i, m, l}\left\langle u_{i}, u_{i}\right\rangle\left\langle v_{l}^{m}, v_{l}^{m}\right\rangle \lambda_{e} \\
& =\sum_{i}{ }_{A}\left\langle u_{i}, u_{i}\right\rangle \sum_{m}\left(\sum_{l}\left\langle v_{l}^{m}, v_{l}^{m}\right\rangle\right) \lambda_{e} \\
& =\mathrm{r}-\operatorname{Ind}[X]\left(\sum_{m} \operatorname{dim}\left(V_{\sigma_{m}}\right) \lambda_{e}\right) .
\end{aligned}
$$

The second statement follows similarly.

We denote by $X \rtimes_{\gamma} V$ the bimodule $\hat{V}$. We call this the crossed product bimodule by bundle $V$.

As in the case of actions on algebras, we need the definition of free actions on bimodules. 
DEFINITION 11 ([KW2]). Let $(X, A, B, \gamma, \alpha, \beta, G)$ be a $G$-equivariant system of bimodules. The action $\gamma$ is called free if for $g \in G, g \neq e$, the following holds:

For $T \in \in_{\mathbb{C}} \operatorname{End}_{\mathbb{C}}(X)$, if

$$
T(a x)=a T(x), \quad T(x b)=T(x) \beta_{g}(b)
$$

for all $x \in X, a \in A$ and $b \in B$, then $T=0$.

We remark that an action $\gamma$ is free if and only if ${ }_{A} X_{B}$ and ${ }_{A}(X g)_{B}$ have no intertwiner for $g \neq e$.

PROPOSITION 12. We assume that the action $\gamma$ of $G$ is free. Then ${ }_{A \rtimes_{\alpha} H}$ End $_{B \rtimes_{B} K}$ $\left(X \rtimes_{\gamma} V\right)$ is isomorphic to elements in ${ }_{A} \operatorname{End}_{B}(X) \otimes\left(\bigoplus_{g \in G} \mathbb{C} \operatorname{End}_{\mathbb{C}}\left(V_{g}\right)\right)$ satisfying $h \cdot T\left(x \otimes v_{g} \lambda_{g}\right)=T\left(h\left(x \otimes v_{g} \lambda_{g}\right)\right)$ and $\left(T\left(x \otimes v_{g} \lambda_{g}\right)\right) \cdot k=T\left(\left(x \otimes v_{g}\right) k\right)$ for $h \in H$, $k \in K, x \in X, g \in G$ and $v_{g} \in V_{g}$.

Moreover, if $X$ is irreducible as an A-B bimodule, the above fixed point subalgebras are isomorphic to ${ }_{H} \operatorname{EndV}_{K}(V)$; if $V={ }_{G} C(G)_{G}$, the above algebra is isomorphic to $\operatorname{End}(X)^{G}$; and if $V={ }_{\{e\}} C(G)_{G}$, isomorphic to $\operatorname{End}(X)$.

PROOF. For $g \in G$, let $\left\{v_{i}^{g}\right\}_{i}$ be a CONS in $V_{g}$. We take the base such that $\left(v_{i}^{g}\right) g^{\prime}=v_{i}^{g g^{\prime}}$. Let $T$ be in ${ }_{A \rtimes_{\alpha} H} \operatorname{End}_{B \rtimes_{\beta} K}\left(X \rtimes_{\gamma} V\right)$. We define $T_{i, j}^{g, g^{\prime}} \in \mathbb{C} \operatorname{End}_{\mathbb{C}}(X)$ as follows.

$$
T\left(x \otimes v_{j}^{g} \lambda_{g}\right)=\sum_{g^{\prime}, i} T_{i, j}^{g, g^{\prime}}(x) \otimes v_{i}^{g^{\prime}} \lambda_{g^{\prime}}
$$

for $x \in X$ and $g \in G$. By the $A-B$ intertwining property of $T$, we have

$$
T_{i, j}^{g, g^{\prime}}(a x)=a T_{i, j}^{g, g^{\prime}}(x), \quad T_{i, j}^{g, g^{\prime}}\left(x \beta_{g}(b)\right)=T_{i, j}^{g, g^{\prime}}(x) \beta_{g^{\prime}}(b) .
$$

Since $\gamma$ is free, if $g \neq g^{\prime}$, we have $T_{i . j}^{g, g^{\prime}}=0$. For $g \in G$, we have $T_{i . j}^{g, g} \in{ }_{A} \operatorname{End}_{B}(X)$. This shows that $T \in{ }_{A} \operatorname{End}_{B}(X) \otimes\left(\bigoplus_{g \in G \mathbb{C}} \operatorname{End}_{\mathbb{C}}\left(V_{R}\right)\right)$.

When $X$ is irreducible ${ }_{A} \operatorname{End}_{B}(X)$ is isomorphic to $\mathbb{C}$, and $T$ can be considered as an element in ${ }_{H} \operatorname{EndV}_{K}(V)$. When $V={ }_{\left({ }_{e} \mid\right.} C(G)_{G}$, each $V_{\mathrm{g}}$ is 1-dimensional. We denote $T^{g}$ by $T_{1,1}^{g . g}$. By $T\left(x \otimes \lambda_{g}\right)=\left(T\left(x \otimes \lambda_{e}\right)\right) g$, we have $T^{g}=T^{e}$ for all $g \in G$. Then $T$ is considered as an element in ${ }_{A} \operatorname{End}(X)_{B}$. When $V={ }_{G} C(G)_{G}$, by the left intertwining property under $G$ of $T, T$ is contained in ${ }_{A} \operatorname{End}_{B}(X)^{G}$.

We state the following categorical structure.

Proposition 13. Let $H, K$ and $L$ be finite subgroups of $G$, and $V={ }_{H} V_{K}, W=$ ${ }_{K} W_{L}$ be bundles over $G$. Let $X={ }_{A} X_{B}$ and $Y={ }_{B} Y_{C}$ be two Hilbert $C^{*}$-bimodules of finite type. Moreover, let $\left(X, A, B, \gamma^{X}, \alpha, \beta, G\right)$ and $\left(Y, B, C, \gamma^{Y}, \beta, \delta, G\right)$ be $G$-equivariant systems of bimodules. Then we have

$$
\left(X \rtimes_{\gamma^{x}} V\right) \otimes_{B \rtimes_{\beta} K}\left(Y \rtimes_{\gamma^{\gamma}} W\right) \simeq\left(X \otimes_{B} Y\right) \rtimes_{\gamma^{x} \not r}\left(V \otimes_{K} W\right)
$$


PROOF. The map $\varphi$ from $(X \rtimes V) \otimes_{B \rtimes_{B} K}(Y \rtimes W)$ to $\left(X \otimes_{B} Y\right) \rtimes_{\gamma} x \otimes r\left(V \otimes_{K} W\right)$ given by

$$
\varphi\left(\left(x \otimes v_{g} \lambda_{g}\right) \otimes\left(y \otimes w_{g^{\prime}} \mu_{g^{\prime}}\right)\right)=\left(x \otimes \gamma_{g}^{Y}(y)\right) \otimes\left(v_{g} \lambda_{g} \otimes w_{g^{\prime}} \mu_{g^{\prime}}\right)
$$

gives the desired isomorphism. Left and right actions are shown to be conserved by computations.

We only show that the left inner products coincide. We compute the left inner product of the left-hand side as follows. We assume $g_{2} g_{2}^{\prime-1}=k \in K$ and $g_{1} g_{2} g_{2}^{\prime-1} g_{1}^{\prime-1}=h \in H$.

$$
\begin{aligned}
A \rtimes_{\alpha} H & \left\langle\left(x \otimes v_{g_{1}} \lambda_{g_{1}}\right) \otimes\left(y \otimes w_{g_{2}} \mu_{g_{2}}\right),\left(x^{\prime} \otimes v_{g_{1}}^{\prime} \lambda_{g_{1}^{\prime}}\right) \otimes\left(y^{\prime} \otimes w_{g_{2}^{\prime}} \mu_{g_{2}^{\prime}}\right)\right\rangle \\
& ={ }_{A \rtimes_{\alpha} H}\left\langle\left(x \otimes v_{g_{1}} \lambda_{g_{1} B \rtimes_{\beta} K}\left\langle y \otimes w_{g_{2}} \mu_{g_{2}}, y^{\prime} \otimes w_{g_{2}^{\prime}}^{\prime} \mu_{g_{2}^{\prime}}\right\rangle, x^{\prime} \otimes v_{g_{1}^{\prime}}^{\prime} \lambda_{g_{1}^{\prime}}\right)\right. \\
& ={ }_{A \rtimes_{\alpha} H}\left\langle\left(x \otimes v_{g_{1}} \lambda_{g_{1}}\right)_{B}\left\langle y, \gamma_{k}^{Y}\left(y^{\prime}\right)\right\rangle\left\langle w_{g_{2}}, k\left(w_{g_{2}^{\prime}}^{\prime}\right)\right\rangle \lambda_{k}, x^{\prime} \otimes v_{g_{1}^{\prime}}^{\prime} \lambda_{g_{1}^{\prime}}\right\rangle \\
& =\left\langle w_{g_{2}}, k\left(w_{g_{2}^{\prime}}^{\prime}\right)\right\rangle_{A \rtimes_{\alpha} H}\left\langle x \beta_{g_{1}}\left({ }_{B}\left\langle y, \gamma_{k}^{Y}\left(y^{\prime}\right)\right\rangle\right) \otimes\left(v_{g_{1}}\right) k \lambda_{g_{1} k}, x^{\prime} \otimes v_{g_{1}^{\prime}} \lambda_{g_{1}^{\prime}}\right\rangle \\
& \left.=\left\langle w_{g_{2}}, k\left(w_{g_{2}^{\prime}}^{\prime}\right)\right\rangle_{A}\left\langle x \beta_{g_{1}}{ }_{B}\left\langle y, \gamma_{k}^{Y}\left(y^{\prime}\right)\right\rangle\right), \gamma_{h}^{X}\left(x^{\prime}\right)\right\rangle\left\langle\left(v_{g_{1}}\right) k, h\left(v_{g_{1}^{\prime}}^{\prime}\right)\right\rangle \lambda_{h} ;
\end{aligned}
$$

otherwise this is equal to zero.

We compute the left inner product of the right-hand side:

$$
A \rtimes_{\alpha} H\left\langle\left(x \otimes \gamma_{g_{1}}^{Y}(y)\right) \otimes\left(v_{g_{1}} \lambda_{g_{1}} \otimes w_{g_{2}} \mu_{g_{2}}\right),\left(x^{\prime} \otimes \gamma_{g_{1}^{\prime}}^{Y}\left(y_{1}^{\prime}\right)\right) \otimes\left(v_{g_{1}^{\prime}}^{\prime} \lambda_{g_{1}^{\prime}} \otimes w_{g_{2}^{\prime}}^{\prime} \mu_{g_{2}^{\prime}}\right)\right\rangle .
$$

We compute the bimodule parts and the bundle parts separately.

At first we compute the bundle part. If $g_{2} g_{2}^{\prime-1}$ is not contained in $K$ the above is zero. We assume $g_{2} g_{2}^{\prime-1}=k \in K$.

$$
\begin{aligned}
{ }_{H}\left\langle\left(v_{g_{1}} \lambda_{g_{1}}\right) \otimes\left(w_{g_{2}} \mu_{g_{2}}\right),\left(v_{g_{1}^{\prime}}^{\prime} \lambda_{g_{1}^{\prime}}\right) \otimes\left(w_{g_{2}^{\prime}}^{\prime} \mu_{g_{2}^{\prime}}\right)\right\rangle & ={ }_{H}\left\langle\left(v_{g_{1}} \lambda_{g_{1} K}\left\langle w_{g_{2}} \lambda_{g_{2}}, w_{g_{2}^{\prime}}^{\prime} \mu_{g_{2}^{\prime}}\right\rangle, v_{g_{1}^{\prime}}^{\prime} \lambda_{g_{1}^{\prime}}\right)\right\rangle \\
& ={ }_{H}\left\langle\left(v_{g_{1}} \lambda_{g_{1}}\right)\left\langle w_{g_{2}}, k\left(w_{g_{2}^{\prime}}^{\prime}\right)\right\rangle \mu_{k}, v_{g_{1}^{\prime}}^{\prime} \lambda_{g_{1}^{\prime}}\right\rangle \\
& =\left\langle w_{g_{2}}, k\left(w_{g_{2}^{\prime}}^{\prime}\right)\right\rangle_{H}\left\langle\left(v_{g_{1}}\right) k \lambda_{g_{1} k}, v_{g_{1}^{\prime}}^{\prime} \lambda_{g_{1}^{\prime}}\right\rangle \\
& =\left\langle w_{g_{2}}, k\left(w_{g_{2}^{\prime}}^{\prime}\right)\right\rangle\left\langle\left(v_{g_{1}}\right) k, h\left(v_{g_{1}^{\prime}}^{\prime}\right)\right\rangle \lambda_{h^{\prime}} .
\end{aligned}
$$

Next, we compute the bimodule part. We put $h=g_{1} g_{2}\left(g_{1}^{\prime} g_{2}^{\prime}\right)^{-1}$. If $h \notin H$, the above is zero. We assume that $h \in H$. When $g_{2} g_{2}^{\prime-1}=k \in K$, we have $g_{1}^{-1} h g_{1}^{\prime}=k \in K$.

$$
\begin{aligned}
{ }_{A}\left\langle x \otimes \gamma_{g_{1}}^{Y}(y), \gamma_{h}^{X \otimes r}\left(x^{\prime} \otimes \gamma_{g_{1}^{\prime}}^{Y}\left(y^{\prime}\right)\right)\right\rangle & \left.={ }_{A}\left\langle x \otimes \gamma_{g_{1}}^{Y}(y), \gamma_{h}^{X}\left(x^{\prime}\right) \otimes \gamma_{h g_{1}^{\prime}}^{Y}\left(y^{\prime}\right)\right)\right\rangle \\
& ={ }_{A}\left\langle x_{B}\left\langle\gamma_{g_{1}}^{Y}(y), \gamma_{h g_{1}}\left(y^{\prime}\right)\right\rangle, \gamma_{h}^{X}\left(x^{\prime}\right)\right\rangle \\
& ={ }_{A}\left\langle x \beta_{g_{1}}\left({ }_{B}\left\langle y, \gamma_{g_{1}}{ }^{-1} h_{g_{1}^{\prime}}\left(y^{\prime}\right)\right\rangle\right), \gamma_{h}^{X}\left(x^{\prime}\right)\right\rangle .
\end{aligned}
$$

If $g_{2} g_{2}^{-1} \in K, g_{1} g_{2}\left(g_{1}{ }^{\prime} g_{2}{ }^{\prime}\right)^{-1} \in H$, both sides coincide by the above calculation; otherwise both sides are zero. 
LEMMA 14. The conjugate bundle $\overline{X \rtimes_{\gamma} V}$ of $X \rtimes_{\gamma} V$ is isomorphic to $\bar{X} \rtimes_{\bar{\gamma}} \bar{V}$ as Hilbert $B \rtimes_{\beta} K-A \rtimes_{\alpha} H$ bimodule, where the conjugate action $\bar{\gamma}$ of $\gamma$ is defined by $\bar{\gamma}_{g}(\bar{x})=\overline{\gamma_{g}(x)}$ for $x \in X$.

PROOF. We define a map $\psi$ from $\overline{X \rtimes_{\gamma} V}$ to $\bar{X} \rtimes_{\bar{\gamma}} \bar{V}$ by

$$
\psi\left(\overline{x \otimes v_{g} \lambda_{g}}\right)=\bar{\gamma}_{g^{-1}}(\bar{x}) \otimes \bar{v}_{g^{-1}} \lambda_{g} \text {. }
$$

Then the two bimodules are isomorphic by $\psi$.

\section{Multiple crossed products}

In this section, we consider a 'crossed product construction' using more than three objects. Let $\Gamma$ be a finite group, $H$ and $K$ be two subgroups of $\Gamma$. Let $V$ be an $H-K$ bundle over $\Gamma$, and $G$ be another finite group.

DEFINITION 15. The pair $(V, G)$ is called a $G$-equivariant system of bundles if the following hold.

(1) $(V, G)$ is a $G$-equivariant system of bimodules when $V$ is considered only as a Hilbert $C^{*}(H)-C^{*}(K)$ bimodule.

(2) $G$ acts on $\Gamma$ as an automorphism group and preserves $H$ and $K$ globally.

(3) The following hold.

(a) $g \cdot(h \cdot v)=(g \cdot h) \cdot(g \cdot v)$, for $g \in G, h \in H, k \in K$ and $v \in V$, and $g \cdot(v \cdot k)=(g \cdot v) \cdot(g \cdot k)$.

(b) $g \cdot V_{\gamma} \subset V_{g \cdot \gamma}$ for $g \in G$ and $\gamma \in \Gamma$.

The $G$ action is automatically unitary from (1).

Let $P, Q$ be two finite subgroups of $G$, and $W$ be a $P-Q$ bundle over $G$.

DEFINITION 16. $(V, W)$ is called a covariant system of hundles if $(V, G)$ is a $G$ equivariant system of bundles.

Let $H, K$ be subgroups of $\Gamma$, let $P, Q$ be subgroups of $G, V$ an $H-K$ bundle over $\Gamma$, and $W$ be a $P-Q$ bundle over $G$. Let $(V, W)$ be a covariant system of bundles. We define the crossed product bundle $V \rtimes W$.

We view $V$ only as a Hilbert $C^{*}(H)-C^{*}(K)$ bimodule and construct the crossed product bimodule $V \rtimes W$. We introduce the bundle structure as follows. The base space is the semi-direct product group $\Gamma \rtimes G$. The fiber space of $(\gamma, g)$ is given by $(V \rtimes W)_{(\gamma, g)}=V_{\gamma} \otimes W_{g}$. Left $(H \rtimes P)$-right $(K \rtimes Q)$ actions are defined by tensor product actions, that is, $h\left(v_{\gamma} \lambda_{\gamma} \otimes w_{g} \lambda_{g}\right)=\left(\left(h v_{\gamma}\right) \lambda_{h \gamma} \otimes w_{g} \lambda_{g}\right)$ and $p\left(v_{\gamma} \lambda_{\gamma} \otimes w_{g} \lambda_{g}\right)=p v_{\gamma} \lambda_{p \gamma} \otimes p w_{g} \lambda_{p g}$. Right actions are defined similarly. We have proven the following lemma. 
LEMMA 17. In this way, $V \rtimes_{\gamma} W$ is made into an $H \rtimes P-K \rtimes Q$ bundle over $\Gamma \rtimes G$.

Let $U=V \rtimes W$, where $V$ is an $H-K$ bundle over $\Gamma$ and $W$ is a $P-Q$ bundle over $G$. Let $T \in{ }_{H \rtimes P} \operatorname{EndV}_{K \rtimes Q}(U)$. Since $T$ commutes with the diagonal algebra $l^{\infty}(\Gamma \rtimes G)$, $T$ is expressed as $T=\sum_{\gamma \in \Gamma, g \in G} f(\gamma, g)$, where $f(\gamma, g) \in \mathbb{C} \operatorname{End}_{\mathbb{C}}\left(V_{\gamma}\right) \otimes_{\mathbb{C}} \operatorname{End}_{\mathbb{C}}\left(W_{g}\right)$. EndV $(U)$ is the set of such $T$ 's which are $H \rtimes P-K \rtimes K$ invariant. We only list the simple cases for the use in Section 4 without proof.

LEMMA 18. (1) Let $(V, G)$ be a $G$-equivariant system of bundles. Then EndV $(V \rtimes G) \simeq \operatorname{End} V(V)^{G}$.

(2) If $V={ }_{\Gamma} V_{\{e\}}$ and $W={ }_{\{e\}} C(G)_{G}$, or $V={ }_{\{e\}} V_{\Gamma}$ and $W={ }_{G} C(G)_{\{e\}}$, then $\operatorname{EndV}(V \rtimes W) \simeq \operatorname{EndV}(V)$.

(3) Let $\Sigma$ be a $G$-orbit in $\Gamma$. We take $\gamma_{0} \in \Sigma$, and $G_{\gamma_{0}}$ to be the stabilizer group at $\gamma_{0}$. Let $V={ }_{\{e\}} C(\Sigma)_{\{e\}}, W={ }_{G} W_{G}$. We define the unitary representation $\pi$ of $G$ on $W_{e}$ by $\pi(g) w_{e}=g w_{e} g^{-1}$. Then $\operatorname{EndV}(V \rtimes W) \simeq \pi\left(G_{\gamma_{0}}\right)^{\prime}$.

Proposition 19. Let $H, K, L$ be subgroups of $\Gamma$ and $P, Q$ and $R$ be subgroups of $G, V_{1}$ be an $H$ - $K$ bundle over $\Gamma, V_{2}$ be a $K-L$ bundle over $\Gamma, W_{1}$ be a $P$ - $Q$ bundle over $G$ and $W_{2}$ be a $Q-R$ bundle over $G$ such that $\left(V_{1}, W_{1}\right)$ and $\left(V_{2}, W_{2}\right)$ be two covariant pairs. Then the two bundles $\left(V_{1} \rtimes W_{1}\right) \otimes\left(V_{2} \rtimes W_{2}\right)$ and $\left(V_{1} \otimes V_{2}\right) \rtimes\left(W_{1} \otimes W_{2}\right)$ are isomorphic.

PROOF. This is the bundle version of Proposition 13. This is already proved at the level of Hilbert $C^{*}(H) \rtimes P-C^{*}(K) \rtimes Q$-bimodules. The fiber of $(\gamma, g)$ in the right-hand side is the direct sum of $\left(V_{\gamma_{1}}^{1} \otimes V_{\gamma_{2}}^{2}\right) \otimes\left(W_{g_{1}}^{1} \otimes W_{g_{2}}^{2}\right)$ such that $\gamma_{1} \gamma_{2}=\gamma$ and $g_{1} g_{2}=g$. The fiber of $(\gamma, g)$ in the left-hand side is the direct sum of $\left(\left(V_{\gamma_{1}}^{1} \otimes W_{g_{1}}^{1}\right) \otimes\left(\left(V_{g_{1}^{-1} \cdot \gamma_{2}}^{2}\right) \otimes W_{g_{2}}^{2}\right)\right.$ 's. They are combined by the map which gives the isomorphism in Proposition 13.

Let $X={ }_{A} X_{B}$ be a Hilbert $C^{*}$-bimodule of finite type, $V$ be a bundle over $\Gamma$, and let $(X, V)$ be a covariant system of bimodules, where $(X, A, B, \varepsilon, \alpha, \beta, \Gamma)$ is the associated $\Gamma$-equivariant system of bimodules.

DEFINITION 20. We say that $G$ conserves the covariant system $(X, V)$ of bimodules if the following are satisfied.

(1) There exists an action $\delta$ of $G$ on $X$, an action $\kappa$ of $G$ on $A$ and an action $\omega$ on $B$ such that $(X, A, B, \delta, \kappa, \omega, G)$ is a $G$-equivariant system of bimodules.

(2) $(V, G)$ is a $G$-equivariant system of bundles.

(3) $G$ actions on $X$ and $V$ satisfy the following: $\delta_{g}\left(\varepsilon_{\gamma}(x)\right)=\varepsilon_{g \gamma} \delta_{g}(x)$ for $g \in G$, $\gamma \in \Gamma$ and $x \in X$, and $\kappa_{g}\left(\alpha_{\gamma}(a)\right)=\alpha_{g \gamma}\left(\kappa_{g}(a)\right)$ for $g \in G, \gamma \in \Gamma$ and $a \in A$ and $\omega_{g}\left(\beta_{\gamma}(a)\right)=\beta_{g \gamma}\left(\omega_{g}(a)\right)$ for $g \in G, \gamma \in \Gamma$ and $b \in B$. 
Proposition 21. Suppose $W$ is a $P-Q$ bundle over $G$ such that $G$ conserves the covariant system $(X, V)$ of bimodules. Then there exist actions $\eta, \zeta$ and $\xi$ of $G$ on the crossed-product bimodule $X \rtimes_{\gamma} V$ such that $\left(X \rtimes_{\gamma} V, A \rtimes_{\alpha} H, B \rtimes_{\beta} K, \eta, \zeta, \xi, G\right.$ ) is also a $G$-equivariant system of bimodules. There exists an action $(\gamma, \eta)$ of $\Gamma \rtimes G$ on $X$ such that $\left(X \rtimes_{\gamma} V\right) \rtimes_{\eta} W$ and $X \rtimes_{(\gamma, \eta)}\left(V \rtimes_{\eta} W\right)$ are isomorphic as Hilbert $\left(A \rtimes_{\alpha} \Gamma\right) \rtimes_{\zeta} P-\left(B \rtimes_{\beta} \Gamma\right) \rtimes_{\xi} Q$-bimodules. Moreover, if the action of $\Gamma \rtimes G$ on $X$ is free, the action of $G$ on $X \rtimes V$ is also free.

PROOF. The first assertion is proved by formal calculations.

$$
\left(x \otimes v_{\gamma} \lambda_{\gamma}\right) \otimes w_{g} \mu_{g} \rightarrow x \otimes\left(v_{\gamma} \lambda_{\gamma} \otimes w_{g} \mu_{g}\right) .
$$

The second assertion is proved as follows. If the action of $\Gamma \rtimes G$ is free, $X(\gamma, g)$ and $X\left(\gamma^{\prime}, g^{\prime}\right)$ have no intertwiner $\gamma \neq \gamma^{\prime}$ or $g \neq g^{\prime}$. Since ${ }_{A}((X \rtimes$ $V) g)_{B} \simeq \sum_{\gamma \in \Gamma} \operatorname{dim}\left(V_{\gamma}\right)_{A}(X((\gamma, g)))_{B}$, if $g \neq e,{ }_{A}((X \rtimes V) g)_{B}$ and ${ }_{A}(X \rtimes V)_{B}$ have no intertwiner. Then ${ }_{A \rtimes_{\alpha} H}(X \rtimes V)_{B \rtimes_{B} K}$ and ${ }_{A \rtimes_{\alpha} H}((X \rtimes V) g)_{B \rtimes_{A} K}$ have no intertwiner. This shows that the action of $G$ on $X \rtimes V$ is free.

This proposition can be considered as the associativity law of crossed products of a bimodule and two bundles.

REMARK. We may state the similar associativity law of crossed products of three bundles.

Let $(X, A, B, \gamma, \alpha, \beta, \Gamma)$ be a $\Gamma$-equivariant system of bimodules, and suppose $G$ conserves this system. Let $V$ be an $H-K$ bundle over $\Gamma$. We assume that the action of $\Gamma \rtimes G$ on $X$ is free, and $X$ is irreducible. We may construct the crossed product bimodule $(X \rtimes V) \rtimes G$. Since $G$ acts on $V, G$ also acts on $\operatorname{End}(V)$ by adjoining $\gamma$.

LEMMA 22. End $\left(\left(X \rtimes_{\gamma} V\right) \rtimes_{\eta} G\right)$ is isomorphic to $\operatorname{EndV}(V)^{G}$.

PROOF. $(X \rtimes V) \rtimes G$ and $X \rtimes(V \rtimes G)$ are isomorphic as bimodules. Since $X$ is irreducible, $\operatorname{End}(X \rtimes(V \rtimes G))$ and $\operatorname{EndV}(V \rtimes G)$ are isomorphic. By Lemma 18, $\operatorname{EndV}(V \rtimes G)$ is isomorphic to $\operatorname{EndV}(V)^{G}$.

\section{Examples}

EXAMPLE 1 . Let $G$ be a finite group, and $(A, X, B, G, \alpha, \gamma, \beta, G)$ a $G$-equivariant system of bimodules. Let $V$ be a finite-dimensional Hilbert space, and $\pi$ a unitary representation of $G$ on $V$. We make two Hilbert $\mathrm{C}^{*}$-bimodules from this data using different methods. 
Let $V \otimes C(G) \simeq \bigoplus_{g \in G} V$ be a $G-G$ bundle given as follows.

$$
\begin{aligned}
& g\left(v_{g^{\prime}} \otimes \lambda_{g^{\prime}}\right)=\pi_{g}\left(v_{g}\right) \otimes \lambda_{g g^{\prime}} ; \\
& \left(v_{g^{\prime}} \otimes \lambda_{g^{\prime}}\right) g=v_{g^{\prime}} \otimes \lambda_{g^{\prime} g^{\prime}} .
\end{aligned}
$$

This definition is given in [KoY]. Here $X \otimes V$ means an $A-B$ bimodule given from the outer tensor product of ${ }_{A} X_{B}$ and ${ }_{\mathbb{C}} V_{\mathbb{C}}[\mathrm{KW} 1]$. Then we may construct the crossed product bimodule $(X \otimes V) \rtimes_{\gamma \otimes \pi} G$ by a group and the crossed product bimodule $X \rtimes_{\alpha}(V \otimes C(G))$ by a bundle. The former is defined in [KW2], and the latter is defined in this paper.

PROPOSITION 23. $(X \otimes V) \rtimes_{\gamma \otimes \pi} G$ and $X \rtimes_{\gamma}(V \otimes C(G))$ are isomorphic as $A \rtimes_{\alpha} G-B \rtimes_{\beta} G$ bimodules.

ProOf. $V$ can be considered as the bundle over the trivial group, and $X \otimes V$ is a trivial example of a crossed product by a bundle. Moreover, $G$ conserves the $\{e\}$-equivariant system $(X, A, B$, id, id, id, $\{e\}) . V \otimes C(G)$ can be considered as the crossed product bundle of $V$ by $C(G)$ over $G$. Then $X \rtimes_{\gamma}(V \otimes C(G))$ is the crossed product bimodule by a bundle. These are isomorphic by Proposition 21 .

EXAMPLE 2. Let $P=\Gamma \rtimes G$ be a semi-direct product group, where $\Gamma$ and $G$ are finite groups. Let $A$ be a unital simple $\mathrm{C}^{*}$-algebra and $\alpha$ a properly outer action of $\Gamma$ on $A$. Put $V={ }_{\{e\}} C(\Gamma)_{\Gamma}$. We construct a crossed product bundle $V \rtimes G$, which is a $G$ - $P$ bundle over $P$. This is the bundle given by the inclusion $G \subset P$. We may compute the structure of the tensor powers of $X=A \rtimes(V \times G)$ and $\bar{X}$ easily using the theory in Section 3.

Proposition 24. The $X \otimes \bar{X} \otimes X \otimes \cdots$ 's are isomorphic to $(A \rtimes(V \otimes \bar{V} \otimes V \otimes$ $\cdots)) \rtimes G$. Moreover the endomorphism algebras $\operatorname{End}(X), \operatorname{End}(X \otimes \bar{X}), \operatorname{End}(X \otimes$ $\bar{X} \otimes X)$ and $\operatorname{End}(X \otimes \bar{X} \otimes X \otimes \bar{X})$ are isomorphic to $\operatorname{EndV}(V)^{G}, \operatorname{EndV}(V \otimes \bar{V})^{G}$, EndV $(V \otimes \bar{V} \otimes V)^{G}$ and $\operatorname{EndV}(V \otimes \bar{V} \otimes V \otimes \bar{V})^{G}$ respectively.

PROOF. This follows from Proposition 21 and Lemma 22.

The above action of $G$ on $A \rtimes V$ is strongly outer in the sense of [CK, KW2].

For $g \in G$ we define a unitary operator $\pi(g)$ on $l^{2}(\Gamma)$ by $(\pi(g) \xi)(\gamma)=\xi\left(g^{-1} \cdot \gamma\right)$. $\pi$ is a unitary representation of $G$ on $l^{2}(\Gamma)$. Let $\left\{\Sigma_{1}=\{e\}, \Sigma_{2}, \ldots, \Sigma_{p}\right\}$ be the family of $G$-orbits in $\Gamma,\left\{\sigma_{1}=e, \sigma_{2}, \ldots, \sigma_{p}\right\}$ be a representative set of each $G$-orbit, $G_{\sigma_{i}}$ be the stabilizing subgroup at $\sigma_{i}$ and $\pi_{\sigma_{i}}$ be the restriction of $\pi$ to $G_{\sigma_{i}}$. Let $m$ be the first integer such that $\pi_{\sigma_{t}}^{\otimes m}$ does not generate any new representation for each $i$. 
LEMMA 25. The bimodule $X$ is of depth $2 m$.

ProOF. We compute the action of $G$ on the end-algebras of the tensor powers $V \otimes \bar{V}, V \otimes \bar{V} \otimes V, \ldots$ etcetera explicitly. For $f \in C(\Gamma)$ and for an integer $n$, we define operators $M_{f}^{n}$ by

$$
M_{f}^{n} \xi\left(\gamma_{1}, \gamma_{2}, \ldots, \gamma_{n}\right)=f\left(\gamma_{1} \gamma_{2} \ldots \gamma_{n}\right) \xi\left(\gamma_{1}, \gamma_{2}, \ldots, \gamma_{n}\right)
$$

for $\xi \in l^{2}(\Gamma)^{\otimes n}$.

We have $\left.{ }_{\{e\}}\left(V \otimes_{\Gamma} \bar{V}\right)_{\{e\}} \simeq \bigoplus_{\gamma \in \Gamma} \mathbb{C} \lambda_{\gamma}, \operatorname{EndV}_{(e\}\}}\left(V \otimes_{\Gamma} \bar{V}\right)_{\{e\}}\right) \simeq l^{\infty}(\Gamma)$. We have also $_{\{e\}}\left(V \otimes_{\Gamma} \bar{V} \otimes_{\{e\}} V\right)_{\Gamma} \simeq{ }_{\{e\}}\left(C(\Gamma) \otimes_{\{e\}} C(\Gamma)\right)_{\Gamma}$. The Endomorphism algebra of this bimodule consists of operators on $B\left(l^{2}(\Gamma) \otimes l^{2}(\Gamma)\right)$ which commute with all $M_{f}^{2}$,s and right translations on the right component. This shows that $\operatorname{EndV}\left({ }_{\{e\}} V \otimes_{\Gamma} \bar{V} \otimes_{\{e\}} V_{\Gamma}\right) \simeq$ $B\left(l^{2}(\Gamma)\right) \otimes I_{l^{2}(G)}$. The isomorphism is given by the Kac-Takesaki operator.

Put $l=2 k$. For the $l$-th tensor power $V \otimes_{\Gamma} \bar{V} \otimes \ldots \otimes_{\Gamma} \bar{V}$, we have

$$
\operatorname{EndV}\left(\{e\}\left(V \otimes_{\Gamma} \bar{V} \otimes \ldots \otimes_{\Gamma} \bar{V}\right)_{\{e\}}\right) \simeq B\left(l^{2}(\Gamma)\right)^{\otimes h-1} \otimes l^{\infty}(G) .
$$

Put $l=2 k+1$. For the $l$-th tensor power $V \otimes_{\Gamma} \bar{V} \otimes \ldots \otimes_{\{e\}} V$, we have

$$
\operatorname{EndV}\left(\{e\}\left(V \otimes_{\Gamma} \bar{V} \otimes \ldots \otimes_{\{e\}} V\right)_{\Gamma}\right) \simeq B\left(l^{2}(\Gamma)\right)^{\otimes k} .
$$

These isomorphisms are given by the following $n$-times Kac-Takesaki operators $W^{n}$ :

$$
W^{n} \xi\left(\gamma_{1}, \gamma_{2}, \ldots, \gamma_{n}\right)=\xi\left(\gamma_{1}, \gamma_{1} \gamma_{2}, \ldots, \gamma_{1} \gamma_{2} \ldots \gamma_{n}\right) .
$$

The tensor power of $\pi$ commute with the $n$-times Kac-Takesaki operator. For $l=$ $2 k+1$, we have

$$
\operatorname{EndV}\left({ }_{\{e\}}\left(V \otimes_{\Gamma} \ldots \otimes_{\{e\}} V\right)_{\Gamma}\right)^{G} \simeq\left(B\left(l^{2}(G)\right)^{8 k}\right)^{\mathrm{ad}\left(\pi^{8 k}\right)} .
$$

The right-hand side is decomposed by the irreducible decomposition of $\pi^{\otimes k}$. For $l=2 k$, we have

$$
\operatorname{EndV}\left({ }_{\{e\}}\left(V \otimes_{\Gamma} \otimes \ldots \otimes_{\Gamma} \bar{V}\right)_{\{e\}}\right)^{\mathrm{ad}\left(\pi^{\otimes k}\right)} \simeq \bigoplus_{i}\left(B\left(l^{2}(\Gamma)\right)^{\otimes k-1}\right)^{\mathrm{ad}\left(\pi_{\sigma_{i}}^{8 k-1}\right)} .
$$

This algebra is decomposed by the irreducible decompositions of the ad $\left(\pi_{\sigma_{i}}^{\otimes k}\right)$ 's. Since $\sigma_{1}=e$, the depth of this bimodule is necessarily even.

In the simplest case, we can write down the inclusions of bimodules more explicitly. We denote by $S_{3}$ the symmetric group of order 3 . This is a semi-direct product of $\Gamma=\mathbb{Z}_{3}$ by $G=\mathbb{Z}_{2}$. Put $X=A \rtimes\left({ }_{[e]} C(\Gamma)_{\Gamma} \rtimes G\right)$. Then the depth of $X$ is 4 by 
Lemma 25. Then the endomorphism algebras $\operatorname{End}(X), \operatorname{End}(X \otimes \bar{X}), \operatorname{End}(X \otimes \bar{X} \otimes X)$ and $\operatorname{End}(X \otimes \bar{X} \otimes X \otimes \bar{X})$ are $\mathbb{C}, \mathbb{C} \oplus \mathbb{C}, \mathbb{C} \oplus M_{2}(\mathbb{C})$ and $\mathbb{C} \oplus M_{2}(\mathbb{C}) \oplus M_{3}(\mathbb{C})$.

We describe the embeddings. We denote $\delta_{g}$ by the Dirac delta function at $g \in \Gamma$ in $C(\Gamma)$. The generator is $V={ }_{\{e\}} C(\Gamma)_{\Gamma}$. The 0 th floor consists only of $\mathbb{C}$. The 1 st floor is $\mathbb{C} \otimes_{\{e\}} V={ }_{\{e\}} C(\Gamma)_{\Gamma}$. The 2 nd floor is as follows. $C(\Gamma) \otimes_{\Gamma} \bar{V} \simeq \mathbb{C} \delta_{e} \oplus\left(\mathbb{C} \delta_{a} \oplus \mathbb{C} \delta_{a^{2}}\right)$. The right-hand side is the $G$-irreducible decomposition. The 3rd floor is as follows. $\left(\mathbb{C} \delta_{a} \oplus \mathbb{C} \delta_{a^{2}}\right) \otimes_{\{e\}} V \simeq\left(\delta_{a}+\delta_{a^{2}}\right) \otimes C(\Gamma)_{\Gamma} \oplus\left(\delta_{a^{2}}-\delta_{a}\right) \otimes C(\Gamma)_{\Gamma}$ The first term is isomorphic to $V$. The second term in the right-hand side appears for the first time at this floor. We call such an irreducible component 'new stuff' ([GDJ]). The 4th floor is as follows. $V \otimes_{\Gamma} \bar{V} \simeq \mathbb{C} \delta_{e} \oplus\left(\mathbb{C} \delta_{a} \oplus \mathbb{C} \delta_{a^{2}}\right)$. On the other hand, $\mathbb{C}\left(\delta_{a}-\delta_{a^{2}}\right) C(\Gamma) \otimes_{\Gamma} \bar{V} \simeq$ $\mathbb{C}\left(\delta_{a}-\delta_{a^{2}}\right) \otimes \delta_{e} \oplus\left(\mathbb{C}\left(\delta_{a}-\delta_{a^{2}}\right) \otimes \delta_{a} \oplus \mathbb{C}\left(\delta_{a^{2}}-\delta_{a}\right) \otimes \delta_{a^{2}}\right)$ The first term is new stuff. The second term $\left.\mathbb{C}\left(\delta_{a^{2}}-\delta_{a}\right) \otimes \delta_{a^{2}}\right)$ is identical to the second term $\left(\mathbb{C} \delta_{a} \oplus \mathbb{C} \delta_{a^{2}}\right)$ in $V \otimes_{\Gamma} \bar{V}$. All irreducible representations of $\mathbb{Z}_{2}$ have appeared. We may draw the graph of derived tower from the above information.

On the other hand the derived tower of the crossed product inclusion $A \subset A \rtimes \Gamma$ is known to be of depth 2 . The graph of this inclusion is of type A. This is the crossed product duality stated in [KW2].

EXAMPLE 3. We compute the special case of the Kac algebra construction by bundle ([KoY, Y2]) using our theory. Let $P, G$ and $\Gamma$ be as in Example 2. Let $V={ }_{\{e\}} \Gamma_{\Gamma}$ and $W={ }_{G} G_{\{e\}}$. Let $A$ be a simple $C^{*}$-algebra and $\alpha$ be a properly outer action of $P$ on $A$. We put $X=(A \rtimes V) \rtimes W$. This is a non-trivial example of the crossed product of a bimodule by a bundle. We construct a crossed product bundle $U=V \rtimes W$. This $U$ is actually ${ }_{\Gamma} P_{G}$ and is shown to be irreducible in [KoY]. By Proposition $21 X \simeq A \rtimes U$. $X$ is an $A \rtimes_{\alpha} \Gamma-A \rtimes_{\alpha} G$ bimodule.

PROPOSITION 26. The bimodule $X$ is of depth 2.

Proof. By Proposition 13, $X \otimes \bar{X}$ is isomorphic to $A \rtimes((V \otimes \bar{V}) \rtimes(W \otimes \bar{W}))$, $X \otimes \bar{X} \otimes X$ is isomorphic to $A \rtimes((V \otimes \bar{V} \otimes V) \rtimes(W \otimes \bar{W} \otimes W)) . V \otimes \bar{V} \otimes V$ is isomorphic to $\bigoplus_{\gamma \in \Gamma\{e\}}\left(\lambda_{\gamma} V\right)_{\Gamma} \simeq|\Gamma| \cdot{ }_{|e|} V_{\Gamma}$, and $W \otimes \bar{W} \otimes W$ is isomorphic to $|G| \cdot{ }_{G} W_{\{e\}}$. Then $((V \otimes \bar{V} \otimes V) \rtimes(W \otimes \bar{W} \otimes W))$ is of the form $|G| \cdot \bigoplus_{i, \gamma \in \Sigma_{i}}\left(\lambda_{\gamma} V\right) \rtimes W$. Since $W={ }_{G} C(G)_{\{e\}}$, by Lemma 18, $\bigoplus_{\gamma \in \Sigma_{i}}\left(\lambda_{\gamma} V\right) \rtimes W$ is isomorphic to the direct $\operatorname{sum}\left|\Sigma_{i}\right| \cdot(V \rtimes W)$. This shows that $X \otimes \bar{X} \otimes X$ is isomorphic to $|P|$-times $V \rtimes W$. Since $V \rtimes W$ is irreducible, $X$ is of depth 2 .

The Kac algebra appears at 2nd floor. We compute $(V \otimes \bar{V}) \rtimes(W \otimes \bar{W})$ and $(\bar{V} \otimes V) \rtimes(\bar{W} \otimes W)$. 
$V \otimes \bar{V}=\bigoplus_{\gamma \in \Gamma} \mathbb{C} \lambda_{\gamma}$ On the other hand, $W \otimes \bar{W}=\bigoplus_{\sigma \in \hat{G} .1 \leq j \leq d_{o}} W_{j}^{\sigma}$, where $W_{j}^{\sigma}=\bigoplus_{1 \leq i \leq d_{\sigma}} C(G) \sum_{g \in G} \bar{\sigma}_{i, j}(g)\left(\bar{\lambda}_{g} \otimes \lambda_{g}\right)$. From these, we have

$$
\begin{aligned}
(V \otimes \bar{V}) \rtimes(W \otimes \bar{W}) & \simeq\left(\bigoplus_{\gamma \in \Gamma} \mathbb{C} \lambda_{\gamma}\right) \rtimes\left(\bigoplus_{\sigma \in \hat{G}}\left(\bigoplus_{1 \leq j \leq d_{\sigma}} W_{j}^{\sigma}\right)\right) \\
& \simeq \bigoplus_{\sigma \in \hat{G}, 1 \leq j \leq d_{\sigma}, i}\left(\bigoplus_{\gamma \in \Sigma_{i}}\left(\mathbb{C} \lambda_{\gamma} \rtimes W_{j}^{\sigma}\right)\right) .
\end{aligned}
$$

The bundle $\bigoplus_{\gamma \in \Sigma}\left(\mathbb{C} \lambda_{\gamma} \rtimes W_{j}^{\sigma}\right)$ is decomposed according to the decomposition of the restriction of $\sigma$ to $G_{\sigma}$ by Lemma 18. The equivalences are described by the representation theory of groups.

On the other hand,

$$
\begin{aligned}
(\bar{V} \otimes V) \rtimes(\bar{W} \otimes W) & \simeq\left(\bigoplus_{\pi \in \hat{\Gamma}, 1 \leq j \leq d_{\pi}} V_{j}^{\pi}\right) \rtimes\left(\bigoplus_{g \in G} \mathbb{C} \lambda_{g}\right) \\
& \simeq \bigoplus_{g \in G, \pi \in \hat{\Gamma}}\left(\bigoplus_{1 \leq j \leq d_{\pi}}\left(V_{j}^{\pi} \rtimes \lambda_{g}\right)\right) .
\end{aligned}
$$

Two bimodules $V_{j}^{\pi} \rtimes \lambda_{g}$ and $V_{k}^{\pi^{\prime}} \rtimes \lambda_{g}^{\prime}$ are isomorphic if and only if $\pi=\pi^{\prime}$ and $g=g^{\prime}$.

If $\Gamma$ is not commutative, these Kac algebras are neither commutative nor cocommutative.

\section{References}

[B] B. Blackadar, $K$-theory for operator algebras, Math. Sci. Res. Inst. Publ. 5 (Springer, Berlin, 1986).

[CK] M. Choda and H. Kosaki, 'Strongly outer actions for an inclusion of factors', $J$. Funct. Anal. 122 (1994), 315-332.

[Co] F. Combs, 'Crossed products and Morita equivalence', Proc. London Math. Soc. 49 (1984), 289-306.

[CMW] R. E. Curto, P. S. Muhly and D. P. Williams, 'Cross products of strongly Morita equivalent C*-algebras', Proc. Amer. Math. Soc. 90 (1984), 315-332.

[GDJ] F. M. Goodman, P. de la Harpe and V. F. R. Jones, Coxeter graphs and towers of algebras, Math. Sci. Res. Inst. Publ. 14 (Springer, Berlin, 1989).

[J] V. Jones, 'Index for subfactors', Invent. Math. 72 (1983), 1-15.

[K] T. Kajiwara, 'Remarks on strongly Morita equivalent $\mathrm{C}^{*}$-crossed products', Math. Japon. 32 (1987), 257-260.

[KW1] T. Kajiwara and Y. Watatani, 'Jones index theory by Hilbert C*-bimodules and K-theory', preprint. 
[KW2] T. Kajiwara and Y. Watatani, 'Crossed products of Hilbert C $C^{*}$-bimodules by countable discrete groups', Proc. Amer: Math. Soc., to appear.

[Kay] T. Kajiwara and S. Yamagami, 'Irreducible bimodules associated with crossed product algebras II', Pacific J. Math. 171 (1995), 209-229.

[KoY] H. Kosaki and S. Yamagami, 'Irreducible bimodules associated with crossed product algebras', Internat. J. Math. 3 (1992), 661-676.

[O] A. Ocneanu, 'Quantized symmetry, differential geometry of finite graphs and classification of subfactors', (Notes by Y. Kawahigashi), University of Tokyo Seminar Notes 45 (1991).

[W] Y. Watatani, Index for $C^{*}$-subalgebras, Memoirs Amer. Math. Soc. 424 (Amer. Math. Soc., Providence, 1990).

[Y1] S. Yamagami, 'A note on Ocneanu's approach to Jones index theory', Internat. J. Math. 4 (1993), 859-871.

[Y2] _ ' 'On Ocneanu's characterization of crossed products', preprint.

[Y3] _ 'Frobinus reciprocity in tensor categories', preprint.

Department of Environmental and Mathematical Sciences

Okayama University

Tsushima, 700

Japan
Graduate School of Mathematics

Kyushu University

Ropponmatsu

Fukuoka, 810

Japan 